\title{
Eine studentische Studie zur kirchlichen Umweltarbeit oder: Schöpfungstheologie im Umweltschutz entdecken
}

\author{
Simone Ziermann \\ Augustana-Hochschule Neuendettelsau
}

Kontakt: simone.ziermann@elkb.de

eingereicht: 28.05.2021; überarbeitet: 03.08.2021; angenommen: 01.09.2021

Zusammenfassung: In dem Text werden Ergebnisse einer Pilotstudie zum kirchlichen Umweltengagement in den Ortsgemeinden vorgestellt. Die Studie wurde im Rahmen einer Lehrveranstaltung als „Forschendes Lehren und Lernen“ durchgeführt und von Studierenden mitverantwortet. Der Erkenntnisgewinn bewegt sich deshalb auf zwei unterschiedlichen Ebenen: Zum einen bewährt sich das Gespräch/Interview als hochschuldidaktische Methode; zum anderen ergibt sich ein inhaltlicher Ertrag zur kirchlichen Umweltarbeit in den Ortsgemeinden: Die Schöpfungstheologie, die dort zugrunde gelegt wird, ist zwar auf den ersten Blick eher eindimensional, auf den zweiten Blick zeigt sich aber ein breites Spektrum von theologischen Fragestellungen und Überlegungen. Hier ließe sich mit religionspädagogischen Angeboten gezielt anknüpfen. Inhaltlich rücken dabei vor allem die theologische Sündenlehre, der kategoriale Bruch zwischen Welt und Eschaton sowie die Bedeutung der Mensch-Tier-Beziehung ins Blickfeld.

Schlagwörter: kirchliche Umweltarbeit, Umweltteams, forschendes Lernen, Tiere

Abstract: This article presents the results from a pilot study concerning the work of environmental teams in local congregations. The study, which was directed by students, took place within the framework of a semester course entitled "Teaching and Learning through Experimentation." Therefore insight could be gained on two different levels. On the one hand, the discussion/interview proves its worth as a didactic method of higher education; on the other hand, it provides relevant gains to the environmental work of the church in the local congregations: at first glance, the Theology of Creation upon which it is based may seem one-dimensional; but at second glance, a broad spectrum of theological questions and considerations begins to emerge. Religious educational activities could be offered around these questions. The central Christian theological concepts addressed are the doctrine of sin, the categorical distinction between World and Eschaton, and the significance of the human-animal relationship.

Keywords: Congregational Environmental Work, Ecology Teams, Learning through experimentation, Animals

\section{Einführung}

„Forschendes Lehren und Lernen: Potentiale und Perspektiven für das kirchliche Umweltengagement" - unter diesem Titel fand im Sommersemester 2020 eine Lehrveranstaltung an der Augustana-Hochschule Neuendettelsau statt (Ziermann, 2019). Leitfragen waren u. a.: Wie sehen die Engagierten die kirchliche Umweltarbeit? Was motiviert sie, sich für die „Bewahrung der Schöpfung“ einzusetzen? Wo lassen sich Stolpersteine erkennen?

Das Ziel des Projektes war ein zweifaches: Zum einen ging es um ein hochschuldidaktisches Anliegen: Studierende sollten die Möglichkeit haben, in die qualitative Forschung als wichtige Methode der Praktischen Theologie hineinzuschnuppern. Zum anderen ging es auf inhaltlicher Ebene darum, das Feld zu diesem aktuellen Thema des kirchlichen Lebens zu sondieren. Mit beiden Anliegen war das Projekt auch als Testlauf gedacht: Wie lässt sich das forschende Lehren und Lernen sinnvoll in die eigene For- 
schung und Lehre integrieren? Und aus welchen Blickrichtungen ist ein genauerer Blick auf die kirchliche Umweltarbeit lohnenswert?

Eine Erkenntnis der Untersuchung lautet: Kirchliche Umweltarbeit und Religionspädagogik können wechselseitig voneinander profitieren, denn in den Erfahrungen der Umweltengagierten klingen viele Aspekte an, die für die Schöpfungstheologie wichtig sind. Die Umweltarbeit vor Ort kann deshalb einerseits für die Religionspädagogik eine Hilfe sein, in der komplexen Gemengelage (Englert, 2018, S. 250) rund um die "Schöpfung“ zielgerichtet dort Schneisen zu schlagen, wo der Schöpfungsglaube für Menschen relevant werden kann. Andererseits bleiben theologische Deutungen in den Interviews oft eher implizit. Die Religionspädagogik kann hier gezielt Angebote machen und helfen, explizit zur Sprache bringen, was die Menschen vor Ort bewegt. So könnten $\mathrm{m}$. E. interessante und gewinnbringende Bildungsprozesse in Gang kommen.

\section{Hochschuldidaktische Rahmenhandlung, Methode und Datenmaterial}

Im Rahmen einer Lehrveranstaltung muss das methodische Vorgehen pragmatisch angepasst werden (Schmidt, 2009, S. 447). Die Darstellungen bei Pirner und Rothgangel (2018) sind dafür Ermutigung und Anregung zugleich.

Im Einzelnen gehen wir folgendermaßen vor: Die Lehrveranstaltung zieht sich in mehreren Blöcken über das ganze Semester (Vorlesungs- und vorlesungsfreie Zeit). Im ersten Schritt tauschen sich die Studierenden zu ihren eigenen Vorerfahrungen und Erwartungen aus (Tribula, 2018, S. 77). Sie lesen sich in die Methoden qualitativer Sozialforschung ein (v. a. Tribula, 2018; Schmidt, 2009) und verschaffen sich anhand einschlägiger Netzwerke einen Überblick über das kirchliche Handlungsfeld (z. B. www.schoepfung-bewahren-konkret.de/; www.ekd.de/agu/; www.frieden-umwelt-pfalz.de/).

Auf dieser Grundlage entwerfen die Studierenden einen Leitfaden für teilstrukturierte Interviews (Tribula, 2018, S. 72-73; Strübing, 2018, S. 102-103), parallel dazu wird der Kontakt mit den Interviewpartner*innen aufgenommen. Die Gesprächspartner*innen werden von der Seminarleitung vermittelt, sie ergeben sich nach dem Schneeballprinzip und auf Empfehlungen aus dem persönlichen Umfeld, in jedem Fall aber so, dass zwischen den Studierenden und den Interviewpartner*innen noch kein persönlicher Kontakt bestand (Tribula, 2018, S. 75-76). Angefragt werden Menschen, die nach eigener Angabe in dem Umweltteam ihrer Kirchengemeinde tätig sind, und zwar sowohl Personen, die sich erst seit kurzem in diesem Bereich engagieren, wie auch langjährig Aktive und eine Person, die auf eine lange Phase der Mitarbeit zurückschaut, gegenwärtig aber eher pausiert.

Insgesamt werden fünf Interviews mit sechs Personen geführt, eins der Interviews ist ein Dreiergespräch mit Interviewerin, dem hauptamtlichen Pfarrer der betreffenden Gemeinde und der Leiterin des dortigen Umweltteams; bei den Interviewten handelt es sich um drei Hauptamtliche und drei Ehrenamtliche. Von den angefragten Personen sagen zwei Frauen und vier Männer zu. Ob sich hier schon abzeichnet, was die Expert*innen mehrfach vermuten, nämlich, dass Umweltarbeit eines der wenigen kirchlichen Handlungsfelder ist, mit denen die Kirche mehr Männer als Frauen anspricht? Das wäre quantitativ zu prüfen.

Großstadt, Kleinstadt, Landgemeinde und Gemeinde im Ballungsraum werden gleichermaßen abgedeckt, der Bezugsrahmen ist bei all dem die Evangelisch-Lutherische Kirche in Bayern.

Die Interviews werden coronabedingt fast ausschließlich per Videokonferenz durchgeführt, aufgezeichnet und anschließend nahe am O-Ton paraphrasiert (Tribula, 2018, S. 76-77). Diesen Paraphrasen sind die folgenden Interviewpassagen entnommen und darauf beziehen sich die angegebenen Belegstellen.

Anschließend werden gemeinsam Kategorien gebildet und die Interviews codiert (Schmidt, 2009, 
S. 448-454). Auf die Möglichkeit einer computergestützten Auswertung wird dabei zwar hingewiesen, in der Kürze der Zeit erfolgt die Auswertung aber „von Hand“.

Parallel zu diesem Prozess führe ich als Seminarleitung Gespräche mit Expert*innen in diesem Bereich, also mit Menschen, die der kirchlichen Umweltarbeit institutionell bzw. von Amts wegen besonders verbunden sind (Strübing, 2018, S. 106-107) und so eine überregionale Perspektive einbringen. Die Gespräche sollen die studentischen Interviews bzw. ihre Auswertung gleichsam flankieren und in den weiteren Diskurs einbetten.

In methodischer Hinsicht ist also klar: Die Studie kann keine repräsentativen Ergebnisse liefern. Sie erfüllt aber das Ziel einer Pilotstudie, nämlich Anhaltspunkte zu geben, wo es sich lohnt, in Zukunft genauer hinzuschauen. In diesem Sinn ist die folgende Darstellung eine Weiterarbeit an der studentischen Auswertung. Sie ist - neben anderen Überlegungen - zugleich die Grundlage, um den Interviewleitfaden für eine anvisierte Folgestudie zu überarbeiten (Tribula, 2018, S. 77; Schmidt, 2009, S. 455).

\section{Ergebnisse}

\section{I Der erste Blick: eine schöpfungstheologische Ernüchterung}

In schöpfungstheologischer Hinsicht fügen sich die Interviews zunächst einmal in das ernüchternde Gesamtbild, das die Religionspädagogik jüngst vom Thema "Schöpfung“ zeichnet (z. B. Hunze, 2018, S. 162-163; Benk, 2018, S. 233). Denn die (mehr oder minder explizite) Gleichsetzung der "Schöpfung“ mit „Natur“ (z. B. Hunze, 2018, S. 168; Steins, 2018, S. 55; Benk, 2018, S. 235; Rothgangel, 2018) und die starke Konzentration auf "die ethischen Implikationen“ (Altmeyer \& Dreesmann, 2018, S. 176) des Schöpfungsglaubens zeichnen sich eben auch in der kirchlichen Umweltarbeit deutlich ab.

Obwohl der Begriff durch den Interviewleitfaden nicht eingetragen wird, verstehen alle Interviewten ihr Engagement für Umwelt- und Klimaschutz als „Bewahrung der Schöpfung“ (z. B. Pm2/1; Pm2/3; Pm4/2; Pm5/2) und diese Deutung durchzieht auch die kirchlichen Netzwerke wie ein roter Faden (z. B. https://umwelt-evangelisch.de).

Zwar besteht eine große Bereitschaft, das konkrete Umweltengagement in der Gemeinde mit geistlichen Angeboten zu verbinden und Schöpfungsgottesdienste sind oft eine feste Größe in der Arbeit der Umweltteams (Pm3/6), allerdings haben auch Andachten und Gottesdienste oft eine erkennbare ethische Stoßrichtung: von der Gebetsnacht anlässlich einer Klimakonferenz oder der Andacht zum Jubiläum des BUND (Pm2/9), bis hin zum Festgottesgottesdienst mit offiziellem Startschuss des Umweltmanagements „Grüner Gockel“ (Pm4/4). Auch in kirchlichen Bildungsangeboten für Umweltengagierte nehmen „Umwelttipps“ (Benk, 2018, S. 246) einen breiten Raum ein (z. B. https://umwelt-evangelisch.de/unsere-termine/weiterbildungangebot) und so scheint die Religionspädagogik in Hinsicht auf die kirchliche Umweltarbeit vor ebenso "gravierenden, schöpfungsspezifischen Herausforderungen" (Hunze, 2018, S. 162-163) zu stehen, wie an anderen Orten religiöser Bildung.

\subsection{Der zweite Blick: Schöpfungstheologie im Umweltschutz entdecken}

Auf den zweiten Blick allerdings stecken vielfältige Aspekte von (Schöpfungs-)Theologie in den Interviews. Denn ob beim Nachdenken über ethische Implikationen oder bei der Frage nach dem Verhältnis von "Genesis“ und Weltentstehung (Rothgangel, 2018), die Rede von der "Schöpfung“ meint vor allem „(Glaubens-)Erfahrung und (Glaubens-)Deutung“ (Hunze, 2018, S. 168). Eben solche (Glaubens-)Erfahrungen lassen sich in den Interviews in unterschiedlichen Facetten erkennen.

\subsection{Umwelt schützen - Lebensfreude erfahren}

In den Interviews wird an vielen Stellen erkennbar: Im kirchlichen Umweltengagement machen Menschen positive und bewegende Erfahrungen. Namentlich gemeinsame Aktionen aller Art sind ein be- 
sonderes Highlight für die Interviewten. Da werden Nistkästen gebaut (Pm4/7), Blühwiesen angelegt und mit Insektenhotels versehen (Pm4/7; Pm4/4), Müllsammelaktionen mit Kindergruppen (Pm4/11) und generationenübergreifendes Umweltfasten (Pm4/7) organisiert. Dieses Erleben wird durchaus auch geistlich-theologisch gedeutet. So verweist ein Pfarrer explizit auf die spirituelle Dimension der Arbeit im Garten:

Pm1/3b: Und man merkt, gerade jetzt in der Corona-Zeit [...], wie gut es mir selbst getan hat, einfach abends im Garten zu arbeiten. Das hat eine Tiefenspiritualität, die nicht zu unterschätzen ist...

Zum Beispiel lässt sich in der Auseinandersetzung mit der Natur viel darüber lernen, wie das Zusammenleben in guter Weise funktionieren könnte:

Pm1/3: [...] Sodass man auch sieht: Es muss nicht immer alles sofort sein, unsere ganze Gesellschaft ist so auf Leistung gedrillt und das geht halt nicht. Die Pflanzen müssen wachsen und brauchen Zeit, sich zu entwickeln und das will gehegt und gepflegt werden und braucht Sorgsamkeit und Achtsamkeit....

Spürbare Begeisterung kommt bei all dem spätestens auf, sobald Tiere ins Spiel kommen (Pw1/3). Wenn zum Beispiel erzählt wird, wie die jungen Falken im Kirchturm via Internetkamera beobachtet werden können (Pm3/4; Pm3/7) oder die "gemeindeeigenen“ Krähen den Schöpfungsgottesdienst „mitgestalten" (Pw1/3a-Pw1/3b). Auch hier werden theologische Deutungen aufgegriffen, so erzählt eine Umweltbeauftragte unmittelbar nach der Schilderung rund um die Krähen im Pfarrgarten:

Pw1/3b: [...] Sondern man hat eher das Gefühl: Hoppla, wir haben da nur einen kleinen Stein reingeschmissen und jetzt zieht das solche Kreise und ich glaub, das ist das, was [...Name wird genannt] mit dem Heiligen Geist meint. Also, den merkt man daran, dass es einfach vorwärtsläuft ohne große Kraftanstrengung. Man erreicht Dinge, die sonst im normalen Leben, im normalen Berufsalltag gar nicht möglich sind, wo man sehr viel mehr Kraftaufwand reinstecken muss, die erreicht man mit Freude und mit Leichtläufigkeit.

\section{Ertrag}

In der Auseinandersetzung mit der Natur und im Engagement für Umweltschutz klingt eben jene „emotionale Tönung“ (Englert, 2018, S. 261) und „Daseinsfreude“ (Englert, 2018, S. 262) an, die rund um den Schöpfungsglauben so wichtig ist. Empathische Mitgeschöpflichkeit (Riede, 2018, S. 86-87) ist immer wieder mit Händen zu greifen.

Die Erfahrungen der Umweltengagierten führen also deutlich vor Augen, wie ethisches Engagement und Glaubensperspektive ineinander übergehen können, und dieser Schnittstelle ließe sich gemeinsam mit den Engagierten genauer nachgehen: Welche Erfahrungen mit Gott und mit dem Glauben machen Menschen, die sich für die „Umwelt" engagieren und davon reden, die „Schöpfung Gottes zu bewahren"? Wie lassen sich die positiven Gemeinschaftserfahrungen theologisch ausdeuten und mit differenzierten geistlichen Angeboten verbinden?

Nicht zuletzt markieren die Erfahrungen der Umweltengagierten aber auch eine wichtige inhaltliche Leerstelle der religionspädagogischen Theoriebildung: Die Mensch-Tier-Beziehung wird in der Religionspädagogik bisher kaum wahrgenommen. Wenn sich in einem Jahrbuch zur "Schöpfung“ nur ein Beitrag dezidiert mit Tieren beschäftigt und dieser deutlich ethisch ausgerichtet ist (Riede, 2018), muss man sich die Frage stellen, ob sich die religionspädagogische Debatte nicht selbst in jener ebenso anthropozentrischen wie ethischen Verengung bewegt, die wiederholt kritisiert wird. Wie kann es gelingen, die hohe emotionale Beteiligung aufzugreifen, die sich mit Tieren verbindet? Hier stehen die Überlegungen, soweit ich sehe, noch ganz am Anfang (Hagencord, 2015; Horstmann, 2018; Naurath, 2018).

\subsubsection{Gleichgülttigkeit und Trägheit deuten}

Trotz aller Bildungsbemühungen - die praktischen Konsequenzen in Sachen Nachhaltigkeit bleiben überschaubar (Altmeyer \& Dreesmann, 2018, S. 171-172). Das Engagement zu verstärken, das Material zu verbessern, an der Öffentlichkeitsarbeit zu feilen u. s. w. mag wichtig und richtig sein, ist aber sicher 
nicht des Rätsels eine Lösung (Englert, 2018, S. 255). Diese Erfahrung machen auch die Umweltengagierten. Sie investieren zum Teil enorm viel Zeit und Herzblut (Pw1/5-Pm1/5) und zeigen dabei eine große Sensibilität für die Menschen vor Ort. Man bemüht sich in einem hohen Maß, Menschen abzuholen, sie wahrzunehmen, mit einzubinden (auch Pm2/8; Pw1/7a; Pw1/8a):

Pw6/23: Man muss schon die Kirchengemeinde mitnehmen. Und man muss das begründen, was man tut. Man darf nicht einfach tun, nach dem Motto "Friss oder stirb!“. Wenn man z. B. eine Grünfläche anlegt, eine Blühfläche, die man nicht mehr mäht, am Friedhof, muss man über Schilder o.̈̈. kundtun, was der Hintergrund ist, sonst kann ich mir vorstellen, dass das für die alte Frau, die da immer unterwegs war, als das immer sauber gemäht war, ein Problem ist. [...] Wenn man das nicht erklärt, fühlen die Leute sich nicht mitgenommen und dann glaub ich schon, dass es Ärger gibt. Dafür gibt es aber auch einen Gemeindebrief - den wollen wir jetzt auch verstärkt mitnutzen - um dort die Maßnahmen, die man vorhat, auch zu erklären.

Gleichwohl und so gut die Öffentlichkeitsarbeit auch sein mag: Neben viel Interesse stoßen Umweltteams eben auch auf viel Desinteresse (Pm4/19; Pm5/14-Pm5/17) und es ist durchaus nicht so, dass die Ehrenamtlichen immer und überall nur so strömen:

Pw6/13: Eine Schwierigkeit ist sicherlich, die Leute zu finden, die sich dann mit engagieren. Grüner Gockel [Umweltmanagement und-zertifikat], das mache ja nicht ich allein, sondern da müssen wir einen Kreis an Mitarbeitern finden. Da bin ich auch gespannt, wie das wird, wenn es mal wirklich soweit ist, ob man da genug Mitstreiter findet.

Das geht so weit, dass kirchliches Umweltengagement in der Gemeinde auch polarisieren kann (auch Pm3/9):

Pm4/13: [...] Und letztes Jahr, als es dieses Volksbegehren ["Rettet die Bienen“] gegeben hat, haben wir zu diesem Thema etwas im Gemeindebrief veröffentlicht. Wir haben nicht dazu aufgefordert zu unterschreiben, sondern wollten lediglich informieren. Daraufhin haben wir starke Beschwerden von einem Landwirt bekommen, der bei uns in der Gemeinde ist.

Dabei gibt es durchaus nicht nur in den Ortsgemeinden Gegenwind. Auch in der Kirche als Ganzes ist Begeisterung und Unterstützung nicht immer die erhoffte (auch Pw6/14a).

Pm2/11: [...] Da gibt es Phasen, in denen man sich mehr Hilfe wünscht.

Zwar klingt in manchen Gesprächen die Hoffnung auf einen Aufbruch und die neue Generation, Fridays for future u. ä. an (Pm5/16). Aber es wird auch darauf hingewiesen, dass das Thema für die Kirchen ja nicht erst seit gestern auf der Agenda steht (Stichwort "Alt-68er“, Pm1/3), und in der langfristigen Perspektive scheint mancher Aufschwung auch schnell wieder zu „verpuffen“ (Pm3/1).

Insgesamt also lässt sich (auch) in der Kirche eine eigentümliche Trägheit in Sachen Nachhaltigkeit beobachten: „Wir kennen den Weg. Aber er ist so mühsam. So, denke ich, geht es vielen bei uns in der protestantischen Kirche. Wir wissen, was richtig ist, wenn es um die Bewahrung der Schöpfung geht. [...] Aber es ist eben nicht so leicht" (http://www.frieden-umwelt-pfalz.de/index.php?id=299, Stand 25.5.2019, dort zitiert nach OKR Dr. Michael Gärtner).

Vor allem bei einer Expertin wird die emotionale Beteiligung deutlich, die damit einhergehen kann: „Die Uhr tickt! Die Bedrohung durch den Klimawandel rückt immer näher - und dennoch geht es so langsam voran, dennoch schauen Menschen weg oder bleiben gleichgültig..." So klingt es mehrfach sinngemäß an - und das kann für Engagierte eine echte Anfechtung sein.

\section{Ertrag}

Die Stärke der christlichen Anthropologie besteht nicht zuletzt darin, dass sie einen ebenso nüchternrealistischen wie wohlwollenden Blick auf die Menschen wirft. Menschen sind nicht nur Ebenbilder Gottes, die für Gerechtigkeit und Frieden kämpfen, sie sind auch Sünder: träge und gleichgültig, gierig und egoistisch. Das alles ist - und das scheint mir der Knackpunkt - conditio humana, lässt sich also letzten Endes durch keine noch so gewiefte Maßnahme aus der Welt schaffen. Diese nüchterne Perspek- 
tive der theologischen Anthropologie dürfte in der Praxis so manchen Frust relativieren, mancher Debatte die Schärfe nehmen und dadurch insgesamt entlasten.

Die Erfahrungen der Umweltteams machen also deutlich: An der Schnittstelle von Schöpfung und Anthropologie (Englert, 2018, S. 262) darf man die Sündenlehre als Deutungsangebot nicht unterschätzen. Das wiederum bedeutet für die Religionspädagogik schlicht und ergreifend, dass sie genau auf der richtigen Spur ist, wenn sie den Schöpfungsglauben theologisch vertieft (Hunze, 2018, S. 164) und „in einen umfassenden theologischen Horizont" (Benk, 2018, S. 233) einbindet. Auf dieser Grundlage können reflektierte Deutungsangebote gemacht werden, die passgenau an die Erfahrungen vor Ort anschließen und zum Beispiel eine tröstende Perspektive eröffnen, wenn die besten Bemühungen doch nicht die erhofften Früchte tragen.

Das gilt auch und umso mehr, weil die Logik von den guten Engagierten und den gleichgültigen Sündern - man ahnt es schon - allzu einfach wäre. So gut das Engagement auch gemeint sein mag: Auch der Einsatz für die „Bewahrung der Schöpfung“ ist ambivalent.

\subsubsection{Ambivalenz aushalten}

Dass die Anthropozentrik rund um die „Bewahrung der Schöpfung“ ein waschechtes Dilemma darstellt, ist bekannt: Der Mensch schützt sich selbst, indem er die Welt vor sich selber schützt (Steins, 2018, S. 58), wer die „Natur" schützen will, müsste den Menschen abschaffen, wer die Welt enkeltauglich halten will, müsste auf Kinder und Enkel verzichten (Loichinger, 2018, S. 100-101) u. ä. Auch diese ambivalenten Facetten der Schöpfungstheologie lassen sich in den Gesprächen nachzeichnen, und zwar auf verschiedenen Ebenen:

Immerhin geht es bei der „Bewahrung der Schöpfung“ vor allem darum, den eigenen Lebensraum zu erhalten. Die Welt soll lebenswert für den Menschen bleiben, vor allem auch für die nachfolgenden Generationen (Pm1/3; Pm3/4; Pm5/2; Pm5/8). Mit anderen Worten: Indem der Mensch sich für die Schöpfung einsetzt - setzt er sich vor allem für sich selber ein. Das sprichwörtliche „die Natur braucht uns nicht, aber wir brauchen die Natur" klingt in den Gesprächen mehrfach an.

Dazu passt, dass die „Natur“ primär in Bezug auf den Menschen wahrgenommen wird. Sie ist die Hintergrundfolie, die Bühne, auf der Menschen zusammenkommen und sich erholen können (Pm1/1; Pm1/4), ihrem Drang nachgehen, „in der Erde zu wühlen und das Grün zu genießen“ (Pm1/1), zu gestalten, zu wirken, sich „sinnvoll“ zu betätigen... Mit anderen Worten: Die „Schöpfung“ und ihre „Bewahrung“ ist immer auch Mittel zum Zweck zur Selbstverwirklichung des Menschen und zur Befriedigung seiner Bedürfnisse.

Dementsprechend ist schöne Natur in den Augen vieler Menschen vor allem (von Menschen) gepflegte und gestaltete Natur (Pm1/3). Wo Blühwiesen u. ä. zu Wildwuchs führen, braucht es gezielte Öffentlichkeitsarbeit, um Akzeptanz zu schaffen (Pm2/8; Pw1/7). Die Engagierten selbst positionieren sich dazu unterschiedlich - über Schönheit lässt sich bekanntlich streiten. In jedem Fall aber wird deutlich: Natur „an sich“ gibt es nicht, sie wird auf die eine oder andere Weise als Gestaltungsraum des Menschen wahrgenommen. Wer die Umwelt "schützt“, „gestaltet“ diese im selben Atemzug. Um es plakativ zu formulieren: Der Mensch, der die Umwelt schützt, ist derselbe homo faber, der sie gefährdet. Ein Interviewpartner bringt das Dilemma auf den Punkt:

F5/30: Wenn Sie träumen dürften, wie sähe Ihre Wunschvorstellung von kirchlichem Umweltengagement aus? [...]

Pm5/30: [...] In der paradiesischen Schöpfung soll der Mensch seinen Platz haben, um zu leben und sich zu freuen, aber nicht die Macht, diese zu zerstören, der jetzige Mensch tut aber eben genau dieses, deshalb müsste ich mir den jetzigen Menschen wegträumen. 
Neben diesen Bereichen, in denen die „Bewahrung der Schöpfung“ eher unterschwellig-latent anthropozentrisch geprägt ist, gibt es auch Bereiche, in denen die Anthropozentrik des Umweltengagements manifest und offensichtlich wird. Das zeigt sich am deutlichsten in der Art und Weise, wie die Engagierten von Tieren berichten - oder eben nicht: Auf der einen Seite sorgen Tiere für Begeisterung (vgl. oben), werden gehegt und gepflegt. Davon sind aber nur bestimmte Gattungen betroffen, nämlich ziemlich genau die "Zielgruppe“ des „Landesbundes für Vogelschutz": Vögel (Pm3/6; Pm3/12), Fledermäuse (Pm2/17) und natürlich Insekten, allen voran die Bienen. Keine Kirchengemeinde, so hat man den Eindruck, ohne das obligatorische Insektenhotel und die dazugehörige Blühwiese (Pm4/4; Pm5/10; Pm5/11, Pm2/8) - auch die Enkelin soll sich schließlich noch an den Schmetterlingen freuen dürfen.

Auf der anderen Seite und gerade deshalb fällt auf, dass andere Tiere nicht im Blickfeld sind, ist von Tiertransporten, Nutztierhaltung und Billigfleisch in keinem einzigen Interview die Rede. Eine Expertin spricht zwar den übermäßigen Fleischkonsum an, bringt ihn aber unmittelbar mit der Abholzung der Regenwälder und der damit verbundenen Gefährdung des Klimas in Verbindung. Diese Beobachtungen bedeuten freilich nicht - das sei hier ausdrücklich betont -, dass Tierwohl für die Gesprächspartner*innen keine Rolle spielt. Es bedeutet aber, dass der Umgang mit Nutztieren bei keinem/keiner der Interviewten so sehr "oben auf“ lag, dass es in den Gesprächen Thema geworden wäre. Das ist besonders auffällig, weil die Produktion und der Umgang mit Lebensmitteln an und für sich durchaus ein großes Thema ist (Pm1/1; Pm1/1; Pm4/11; Pm2/4). Dabei sind aber offensichtlich vor allem pflanzliche Lebensmittel im Blick, denen man beim „Wachsen und Reifen“ (Pm1/3) zusehen kann. „Bewahrung der Schöpfung“ scheint also im engeren Sinn „Umwelt- und Klimaschutz“ zu bedeuten. Tiere werden wahrgenommen, sofern sie für dieses Anliegen eine Rolle spielen (Insekten, Artenvielfalt), oder den Menschen dabei zufällig „„über den Weg laufen“ (die Krähen im Garten).

\section{Ertrag}

So erhärtet sich zunächst einmal der Befund: Tiere gehören ins Zentrum der Aufmerksamkeit! Nicht nur, aber eben auch in ethischer Perspektive (Benk, 2018, S. 244-245; Riede, 2018; Sajak, 2015, S. 70-71).

Davon abgesehen ist die Selbstbezogenheit des Menschen aber latent allgegenwärtig und als solches unausweichlich. Die Theologie hat auch für diese Fragestellung (mindestens) eine tragfähige Deutung in petto: Menschen sind eben immer beides: simul iustus et peccator. Eine „Bewahrung der Schöpfung“, die sich eindeutig positiv inszeniert und meint, der allgemeinen Ambivalenz entgehen zu können, wäre ein klassisches "gutes Werk", folglich ein Akt der Selbstrechtfertigung - und damit wiederum die Sünde des homo incurvatus, der sich am eigenen Schopf aus dem Schlamassel ziehen will.

Diese Einsicht nimmt jeder Schwarz-Weiß-Logik den Wind aus den Segeln und ist so ein gutes „Hilfsmittel“, wenn in der Kirchengemeinde das Engagement der einen zum Stein des Anstoßes für die anderen wird.

Mit welchen Bildungsangeboten lässt sich an dieser Schnittstelle von Schöpfung und Anthropologie bei den Erfahrungen der Umweltengagierten anknüpfen? Meine Vermutung ist, dass hier die große Stunde der geistlichen Angebote schlägt: eine Andacht einmal für das Umweltteam - und nicht vom Umweltteam für andere, Zeit lassen für Klage (Steins, 2018, S. 57), die ganz bewusst nicht in den Appell zu Engagement und mehr Verantwortung mündet, dem Kummer über die eigenen Grenzen Raum geben, an die man immer wieder stößt, vielleicht mit Abendmahl und der Gelegenheit, mit Gott und mit sich selbst versöhnt zu werden.

Die Möglichkeit, auf spiritueller Ebene mit Herausforderungen umzugehen, ist das große Pfund der kirchlichen Umwelt- und Bildungsarbeit im Unterschied zu allen anderen Akteuren in diesem Bereich und die Umweltteams haben grundsätzlich eine hohe Offenheit für geistliche Formate aller Art (Pm2/9; Pm2/5; Pw1/1; Pm4/4; Pm2/5; Pm3/6; Pm2/9). 


\subsubsection{Mit Trostlosigkeit umgehen}

In der Religionspädagogik wird zum Teil die Tendenz gesehen, die Natur zu romantisieren (Benk, 2018, S. 242) und die „Nachtseiten der Schöpfung“ (Steins, 2018, S. 57) bei der Bildungsarbeit auszublenden. Und tatsächlich erzählen auch die Umweltengagierten vor allem von den schönen und erfreulichen Erlebnissen in und mit der "Schöpfung“ (vgl. oben). Umso mehr sticht eine Interviewpassage heraus und dabei wiederholt sich ein weiteres Mal der Eindruck: Tiere markieren dort, wo sie auftauchen (oder durch Abwesenheit glänzen), Schlüsselstellen.

Pm3/8: Die Dohlen sind mit Sicherheit noch da, aber die Turmfalken werden es nicht geschafft haben. Das

Dohlen-Projekt haben wir vor ungefähr 3-4 Jahren gestartet und die Dohlen haben um den Nistkasten des Turmfalken gekämpft. Das habe ich teilweise sehr hautnah miterlebt und ja, ich muss gestehen, dass ich da manchmal selbst eingegriffen habe, um dem Turmfalken seinen Nistkasten zu erhalten, obwohl das etwas widernatürlich war. Aber mir hat der Falke leidgetan und für die Dohlen hatten wir im Umfeld noch mehr Nistkästen aufgebaut.

In der "zoologischen Schreckenskammer“ (Taxacher, 2018, S. 231) tierischer Grausamkeiten kommt diese Episode noch relativ harmlos daher und doch: „Ungerechtigkeit“ und Gewalt unter Tieren zu beobachten, geht Menschen an die Nieren! Nicht einzugreifen ist manchmal fast unmöglich - auch wenn man es eigentlich natürlich besser weiß. Auch hier schlägt die Empathie (vgl. oben) eben voll zu Buche.

\section{Ertrag}

Wenn Menschen sich mit den bedrückenden Seiten der Natur auseinandersetzen (oder ihnen in einer Unterrichtseinheit ausgesetzt werden), können sie in eine ganze Welle von Emotionen geraten: Wut, Ohnmacht, Traurigkeit... (Horstmann, 2018, S. 89). Solche Erfahrungen lassen Menschen in der Regel (und sinnvollerweise) nur dann zu, wenn sie die Gewissheit haben, dass sie dabei zuverlässig aufgefangen werden. Welche „Ausrüstung“, welche Deutungsangebote hat die Theologie für diesen Fall parat? Der Hinweis auf die Verantwortung des Menschen (z. B. Englert, 2018, S. 259) stößt hier offenkundig an seine Grenzen. Man mag sich noch so sehr engagieren, spätestens beim Versuch, den eschatologischen Tierfrieden herzustellen, beißt sich der Mensch die Zähne aus. Wie soll man sich und andere also trösten, wenn man nicht vertrösten will, aber auch nicht ausblenden kann, dass die Welt ab einem gewissen Grad untröstlich ist.

Damit ergibt sich aus den Erfahrungen der Umweltengagierten - und zwar sowohl aus denen, von denen erzählt wird, als auch aus denen, die manchmal vielleicht ausgeblendet werden - auch in diesem Fall eine Akzentsetzung bei der weiteren theologischen Profilierung des Schöpfungsglaubens (Englert, 2018, S. 262), und zwar in eschatologischer Hinsicht (z. B. Englert, 2018, S. 259): Gefragt ist nicht nur die eschatologische Hoffnung an und für sich. Zur Debatte steht, dass diese eschatologische Hoffnung einen radikalen Bruch impliziert. Wie die "Schöpfung", so handelt eben auch die biblische Rede von der "Erlösung" "nicht von der Welt, die es gibt und die wir erleben“ (Steins, 2018, S. 55 [Hervorhebung im Original]). Sie handelt vielmehr (auch) von einer Welt, die dem menschlichen Handeln kategorial entzogen ist und von Gott selbst realisiert wird.

Damit ist sicher ein Knackpunkt erreicht: Wo ist die Grenze zwischen Trost und Vertröstung? Wo kann und muss verstärktes Engagement eingefordert werden? Und wann wird das Engagement zu verzweifeltem Aktionismus? Für die konkrete Bildungsarbeit ist vermutlich viel gewonnen, wenn es gelingt, die Fragen offen und die Spannungen auszuhalten.

\subsection{Fazit}

Inhaltlich lässt sich zusammenfassen: Einerseits scheint für kirchliche Umweltarbeit über weite Strecken die Gleichung „Umweltschutz gleich Bewahrung der Schöpfung“ zu gelten. Sie ist damit einer Schöpfungstheologie verhaftet, die sich trefflich hinterfragen lässt. In den Erfahrungen der Menschen klingt 
andererseits aber ein facettenreiches Bild von Schöpfungsglauben an. Profilierte religionspädagogische Angebote können helfen, dieses Bild noch klarer und eindrücklicher zu machen.

Die Schwierigkeit dürfte dabei vor allem darin bestehen, dass jeder und jede immer wieder Gratwanderungen ausbalancieren muss: Die Schönheit der Schöpfung wahrnehmen - aber die Augen nicht vor den hässlichen Seiten der Natur verschließen; sich nicht allzu leicht vertrösten lassen - und dennoch getröstet sein, weil von Gott mehr zu erwarten ist als das Menschenmögliche; sich mit vollem Einsatz engagieren - und immer wieder die Ruhepause der geistlichen Besinnung suchen. Religionsunterricht, Konfiarbeit \& Co können für diesen Balanceakt zuverlässige Hilfestellungen geben und dadurch zeigen, wie relevant das christliche Weltbild für ganz alltägliche Erfahrungen sein kann.

Vor diesem Hintergrund sind die Ergebnisse der Studie m. E. auch für das weitere methodische Vorgehen interessant: „[F]achliche Defizite“ (Englert, 2018, S. 255) zu beheben und die Frage, wie „die Aneignung eines differenzierten Schöpfungsbegriffs" (Rothgangel, 2018, S. 125) gelingen kann, ist zweifellos wichtig. Wichtig ist aber auch, induktiv vorzugehen und sehr genau zu erkunden, was Menschen bewegt, wenn sie sich für die „Bewahrung der Schöpfung“ einsetzen.

\section{Zurück zur Rahmenhandlung: hochschuldidaktisches Fazit}

In hochschuldidaktischer Hinsicht verbuche ich das Projekt als Erfolg. Obwohl der methodische Zugang aus sozialwissenschaftlicher Perspektive sicher sehr niederschwellig ist, konnten die Studierenden m. E. einen guten Einblick bekommen, was es bedeuten kann, qualitativ empirisch zu forschen.

Die Mahnung, den Arbeitsaufwand nicht zu unterschätzen (Tribula, 2018, S. 91), kann ich nur unterstreichen. Eine sinnvolle Spur scheint mir, einen klaren Schwerpunkt zu setzen, nämlich entweder auf die Durchführung oder auf die Auswertung von Interviews. In einer Lehrveranstaltung, die methodisch ähnlich angelegt war, konnte zum Beispiel die Transkription der Interviews durch eine Kooperation ausgelagert werden und die Studierenden für die Auswertung unmittelbar an den transkribierten Texten arbeiten. Diese Variante hat sich gut bewährt.

Insgesamt ist für mich in hochschuldidaktischer Hinsicht das Gespräch mit Menschen aus der kirchlichen und gemeindlichen Praxis neu ins Zentrum der Aufmerksamkeit gerückt. Sie sind nicht nur wichtiges Instrument praktisch-theologischer Forschung, sondern auch sinnvolle Methode für die praktischtheologische Lehre. Gespräche mit Menschen vor Ort können die Brücke schlagen zwischen Forschung und Lehre und zugleich ein Brückenpfeiler sein zwischen zeitaufwändigen Praktika auf der einen Seite und dem (allzu) theoretischen Studium auf der anderen Seite.

\section{Literaturverzeichnis}

Altmeyer, Stefan \& Dreesmann, Daniel (2018). Grenzgänge zwischen Natur und Schöpfung: Grundlagen und Vorschläge für fächerverbindendes Lernen in Biologie- und Religionsunterricht. In Stefan Altmeyer, Rudolf Englert, Helga Kohler-Spiegel, Elisabeth Naurath, Bernd Schröder \& Friedrich Schweitzer (Hg.), Jahrbuch der Religionspädagogik: Schöpfung (S. 171-183). Göttingen: Vandenhoeck \& Ruprecht.

Benk, Andreas (2018). „Schöpfung“: trivialisiert, separiert, historisiert und instrumentalisiert - oder eingebunden in den befreienden Horizont biblischer Hoffnung? Kritische Sichtung unterrichtspraktischer Materialien zur Schöpfungsthematik. In Stefan Altmeyer, Rudolf Englert, Helga Kohler-Spiegel, Elisabeth Naurath, Bernd Schröder \& Friedrich Schweitzer (Hg.), Jahrbuch der Religionspädagogik: Schöpfung (S. 229-248). Göttingen: Vandenhoeck \& Ruprecht.

Englert, Rudolf (2018). „Schöpfung“ im Religionsunterricht - eine etwas ernüchternde Zwischenbilanz. In Stefan Altmeyer, Rudolf Englert, Helga Kohler-Spiegel, Elisabeth Naurath, Bernd Schröder \& 
Friedrich Schweitzer (Hg.), Jahrbuch der Religionspädagogik: Schöpfung (S. 250-263). Göttingen: Vandenhoeck \& Ruprecht.

Hagencord, Rainer (2015). Zum Mensch-Tier-Verhältnis im anthropologischen und schöpfungstheologischen Diskurs. Oder: Was christliche Theologie wieder entdecken[sic!] müsste! In Clauß Peter Sajak (Hg.), Schöpfung: Wissen - Kompetenzen - Haltungen. Sekundarstufen I und II (Heft 4) (S. 31-36). Paderborn: Schöningh.

Horstmann, Simone (2018). Das Ich im Anderen: Die Nähe zwischen Kindern und Tieren. In Simone Horstmann, Thomas Ruster \& Gregor Taxacher (Hg.), Alles, was atmet: Eine Theologie der Tiere (S. 7998). Regensburg: Verlag Friedrich Pustet.

Hunze, Guido (2018). „Ich widerspreche alles[sic!], weil eigentlich überall Gott drinsteht.“ Theologische Herausforderungen und schöpfungsdidaktische Stolpersteine (nicht nur für den Religionsunterricht). In Stefan Altmeyer, Rudolf Englert, Helga Kohler-Spiegel, Elisabeth Naurath, Bernd Schröder \& Friedrich Schweitzer (Hg.), Jahrbuch der Religionspädagogik: Schöpfung (S. 161-170). Göttingen: Vandenhoeck \& Ruprecht.

Loichinger, Alexander (2018). Schöpfungsglaube im Anthropozän? In Stefan Altmeyer, Rudolf Englert, Helga Kohler-Spiegel, Elisabeth Naurath, Bernd Schröder \& Friedrich Schweitzer (Hg.), Jahrbuch der Religionspädagogik: Schöpfung (S. 96-108). Göttingen: Vandenhoeck \& Ruprecht.

Naurath, Elisabeth (2018). Werte-Bildung auf dem Erlebnisort Bauernhof. In Stefan Altmeyer, Rudolf Englert, Helga Kohler-Spiegel, Elisabeth Naurath, Bernd Schröder \& Friedrich Schweitzer (Hg.), Jahrbuch der Religionspädagogik: Schöpfung (S. 207-217). Göttingen: Vandenhoeck \& Ruprecht.

Pirner, Manfred L. \& Rothgangel, Martin (Hg.) (2018). Empirisch forschen in der Religionspädagogik: Ein Studienbuch für Studierende und Lehrkräfte. Stuttgart: Kohlhammer.

Riede, Peter (2018). Mitgeschöpflichkeit: Biblische Impulse für eine Tierethik angesichts der Herausforderungen des 21. Jahrhunderts. In Stefan Altmeyer, Rudolf Englert, Helga Kohler-Spiegel, Elisabeth Naurath, Bernd Schröder \& Friedrich Schweitzer (Hg.), Jahrbuch der Religionspädagogik: Schöpfung (S. 85-95). Göttingen: Vandenhoeck \& Ruprecht.

Rothgangel, Martin (2018). Schöpfung und Evolution - eine Beziehung voller Missverständnisse. In Stefan Altmeyer, Rudolf Englert, Helga Kohler-Spiegel, Elisabeth Naurath, Bernd Schröder \& Friedrich Schweitzer (Hg.), Jahrbuch der Religionspädagogik: Schöpfung (S. 123-134). Göttingen: Vandenhoeck \& Ruprecht.

Sajak, Clauß Peter (Hg.) (2015). Schöpfung: Wissen - Kompetenzen - Haltungen. Sekundarstufen I und II. (Heft 4). Paderborn: Schöningh.

Schmidt, Christiane (2009). Analyse von Leitfadeninterviews. In Uwe Flick, Ernst von Kardorff \& Ines Steinke (Hg.), Qualitative Forschung: Ein Handbuch (7. Auflage) (S. 447-456). Reinbek bei Hamburg: Rowohlt-Taschenbuch-Verlag.

Steins, Georg (2018). Alles in bester Ordnung: Ein Interpretationsvorschlag zu den Schöpfungstexten am Anfang der Bibel. In Stefan Altmeyer, Rudolf Englert, Helga Kohler-Spiegel, Elisabeth Naurath, Bernd Schröder \& Friedrich Schweitzer (Hg.), Jahrbuch der Religionspädagogik: Schöpfung (S. 45-59). Göttingen: Vandenhoeck \& Ruprecht.

Strübing, Jörg (2018). Qualitative Sozialforschung: Eine komprimierte Einführung (2. Auflage). Berlin: De Gruyter.

Taxacher, Gregor (2018). Die Mücken des Makarios: Meditation über das Ungeziefer. In Simone Horstmann, Thomas Ruster \& Gregor Taxacher (Hg.), Alles, was atmet: Eine Theologie der Tiere (S. 226-240). Regensburg: Verlag Friedrich Pustet.

Tribula, Christa (2018). Interview. In Manfred L. Pirner \& Martin Rothgangel (Hg.), Empirisch forschen in der Religionspädagogik: Ein Studienbuch für Studierende und Lehrkräfte (S. 71-93). Stuttgart: Kohlhammer.

Ziermann, Simone (2019). Potentiale und Perspektiven für das kirchliche Umwelt- und Klimaengagement. Eine studentische Pilotstudie, augustana Journal, 5, 12-14. 